livraisons

d'Histoire

de l'Architecture

\section{Livraisons de l'histoire de l'architecture}

$23 \mid 2012$

Varia I

\title{
La patrimonialisation du Maroc, entre tradition et rupture de l'héritage français
}

The patrimonialization of Morocco, between tradition and rupture of French heritage

Die Errichtung des Tunnels der Oudayas, eine besondere Initiative des

Denkmalschutzes in Rabat

\section{Nadège Theilborie}

\section{OpenEdition}

\section{Journals}

Édition électronique

URL : http://journals.openedition.org/lha/130

DOI : 10.4000/lha.130

ISSN : 1960-5994

Éditeur

Association Livraisons d'histoire de l'architecture - LHA

Édition imprimée

Date de publication : 15 juin 2012

Pagination : 115-119

ISSN : 1627-4970

Référence électronique

Nadège Theilborie, "La patrimonialisation du Maroc, entre tradition et rupture de l'héritage français », Livraisons de l'histoire de l'architecture [En ligne], 23 | 2012, mis en ligne le 15 juin 2014, consulté le 19 avril 2019. URL : http://journals.openedition.org//ha/130 ; DOI : 10.4000//ha.130

Ce document a été généré automatiquement le 19 avril 2019

Tous droits réservés à l'Association LHA 


\title{
La patrimonialisation du Maroc, entre tradition et rupture de l'héritage français
}

\author{
The patrimonialization of Morocco, between tradition and rupture of French \\ heritage \\ Die Errichtung des Tunnels der Oudayas, eine besondere Initiative des \\ Denkmalschutzes in Rabat
}

Nadège Theilborie

\section{L'exemple de la Kasbah des Oudayas à Rabat}

1 La période du protectorat français (1912-1956) a marqué le Maroc en matière de patrimoine et d'urbanisme notamment grâce à la politique de son premier résident général, Hubert Lyautey (1854-1934). En effet, ce dernier est l'investigateur au Maroc d'un véritable modèle de patrimonialisation. Tout au long de son mandat, Hubert Lyautey a mis en place la première politique culturelle du pays. De fait, dans les premières années du protectorat, un ensemble législatif se tisse afin de protéger le patrimoine marocain. Dès le 26 novembre 1912, un dahir relatif à la conservation des monuments et inscriptions historiques voit le jour ${ }^{1}$. Ce décret est réalisé dans le but de prévenir au mieux des destructions faites par les colons. Cette mesure de prévention peut se comprendre dans la mesure où l'épisode algérien avait marqué le résident général. Parallèlement, est créée une administration ${ }^{2}$ en charge d'établir une liste de classement des monuments historiques du Maroc, confiée à l'artiste Maurice Tranchant de Lunel (1869-1944). Dès le 13 février 1914, un nouveau dahir relatif à la conservation des monuments historiques ${ }^{3}$ est promulgué. Ce dernier, plus complet que le précédent, fonde une véritable politique de conservation du patrimoine marocain, avec notamment la création de zones de protection autour des sites pittoresques et des monuments historiques. Le classement est 
alors confié au service des Antiquités, Beaux-arts et Monuments historiques. Le 6 juin 1914, la Kasbah des Oudayas de Rabat devient le premier monument historique officiel du Maroc $^{4}$.

2 La Kasbah des Oudayas, symbole de l'âge d'or de la dynastie almohade fut construite par Abd al- Mumin (1147-1163) en 1150, et fut délaissée par ses successeurs et ce, jusqu'au XVII ${ }^{\mathrm{e}}$ siècle. En effet, elle fut réhabilitée par les Mauresques chassés d'Espagne par le roi Philippe II, à partir de 1609. Ces derniers créant même une république autonome. Néanmoins, lorsque la dynastie alaouite pris le pouvoir en 1666, le sultan Moulay Rashid (1666-1672) entama la construction d'une résidence princière à l'intérieur de la Kasbah des Oudayas. Cette forteresse construite sur le flanc d'un rocher surplombe l'embouchure du Bouregreg et nous offre un panorama exceptionnel sur l'océan Atlantique et la ville de Salé. En 1914, des travaux de restaurations furent exécutés pour redonner à la Kasbah sa splendeur. Maurice Tranchant de Lunel et son équipe décidèrent de restaurer la porte monumentale de la forteresse - Bab al Kebir - joyaux de l'art almohade. La grande porte avait été murée par les habitants de la Kasbah et transformée en prison au XVIII ${ }^{e}$ siècle, sous le joug de la dynastie alaouite. Les Archives de la Bibliothèque nationale du royaume du Maroc (ABNRM) à Rabat nous permettent d'étudier l'histoire de la restauration de cette porte. En effet, nous apprenons que le service des Antiquités, Beaux-arts et Monuments historiques travaillait avec plusieurs maalem ${ }^{5}$ marocains, dont le plus mentionné dans les archives est Hadj Driss Tourouguy. Ce dernier travailla sur tous les chantiers de la ville de Rabat, aussi bien à la tour Hassan que dans la nécropole de Chellah. Hadj Driss Tourouguy accompagné de son équipe d'ouvriers, désencombra la porte de la Kasbah en décembre $1914^{6}$. À l'intérieur, les travaux s'appliquèrent à restaurer exclusivement les voûtes, entièrement reconstruites par l'entrepreneur Jean-Baptiste David qui acheva les travaux en septembre 1918. Le chantier de restauration se poursuivit avec le réaménagement de la médersa des Oudayas et le jardin andalou. En effet, à partir de 1915, le patio de la médersa fut aménagé par le maalem Mohamed Bel Hadj . Dès 1916, un jardin andalou remplaçait les friches qui entouraient la médersa des Oudayas. À partir de 1925, une fois le gros œuvre achevé, les restaurations de la Kasbah portèrent sur l'enceinte, les remparts, les bastions et les murs de soutènement. La restauration de la Kasbah des Oudayas est l'un des plus grands chantiers de la ville de Rabat et le pouvoir politique pense en faire la publicité. Il fut en effet question de réaliser une inscription aux pieds de la porte monumentale almohade mentionnant la nature et l'ampleur des travaux effectués par le premier résident général Hubert Lyautey et le chef du service des Antiquités Beaux-arts et Monuments historiques, Maurice Tranchant de Lunel ${ }^{8}$. La Kasbah des Oudayas accédait pleinement au rang de véritable objet patrimonial et ce, jusqu’à maintenant.

\section{Le développement économique et la question du patrimoine, contrainte ou atout : le cas du tunnel de la Kasbah des Oudayas}

3 En 1956, Mohammed V (1927-1961) devient le premier roi de l'indépendance et conserve la plupart des lois patrimoniales instaurées par le gouvernement du protectorat. Par ailleurs, dans les années 1980-1990 le Maroc développe des notions telles que «le patrimoine naturel» ou «le patrimoine oral». Car la majorité des monuments 
historiques actuels au Maroc furent inscrits durant la période du protectorat français. Cependant, les bâtiments inscrits depuis l'indépendance regroupent des édifices plus récents, tels que des immeubles publics ou privés, datant de la période du protectorat ou encore des espaces verts comme le jardin d'essai de Rabat, inscrit depuis le 6 mars $1992^{9}$. En outre et ce, depuis quelques années, le développement économique du Maroc s'accélère rapidement, ce qui entraîne une politique de grands travaux dans le pays. En effet, sous l'égide de Mohammed VI, roi depuis 1999, la ville de Rabat et sa voisine Salé reçoivent un nouveau plan d'aménagement urbain. En 2001, le roi fit appel à un groupe de spécialistes comprenant des ingénieurs, des urbanistes, des architectes, des géographes, des économistes, des historiens ainsi que des paysagistes pour établir un projet d'infrastructures publiques de grande envergure dans la vallée du Bouregreg. De plus des bureaux d'études nationaux vérifient la faisabilité du projet d'aménagement. C'est pourquoi le 23 novembre 2004, le projet d'aménagement global de la vallée du Bouregreg est présenté au roi Mohammed VI. Ce projet est désormais dans les mains de l'Agence d'aménagement du Bouregreg qui a été spécialement créé le 23 novembre 2005 afin de réaliser le plan d'urbanisme. Le lancement officiel des travaux est annoncé le 7 janvier 2006. Ce projet d'aménagement compte plusieurs infrastructures dont la réalisation d'un tramway allant de la ville de Salé à la ville de Rabat, la construction du pont Hassan II, mais également la mise en place d'un tunnel sous la Kasbah des Oudayas afin de dégager le trafic routier de la rive de Rabat. Néanmoins, rappelons que la vallée du Bouregreg est dotée de splendeurs du patrimoine architectural marocain, dont la tour Hassan, la nécropole de Chellah, la Kasbah des Oudayas, mais aussi la médersa de Salé et la grande porte Bab Lamrissa. Il se pose donc l'éternel problème de la modernisation de la ville au détriment des vestiges du passé. C'est pourquoi nous nous sommes intéressés particulièrement à la création du tunnel sous les Oudayas.

4 En effet, ce tunnel d'un kilomètre de long est ouvert à la circulation depuis mai 2011. Comment un tunnel a pu être construit sous le premier monument historique du Maroc? La zone de protection autour des monuments historiques n'est-elle plus d'actualité ? Il est vrai qu'après de nombreuses recherches, il nous est apparu que peu de voix, même quasiment aucune, ne se sont élevées contre la construction du tunnel des Oudayas. Il existe pourtant des associations au Maroc qui militent contre la destruction d'un patrimoine architectural, comme l'association Casamémoire. Force est de constater que les deux associations connues à Rabat l'association Ribath el Fath et l'association du Bouregreg ont collaboré au plan d'aménagement de la vallée, d'après le projet d'aménagement de la vallée du Bouregreg ${ }^{10}$. Est-il alors possible que la construction du tunnel sous la Kasbah des Oudayas n'ait pas été un problème d'ordre patrimonial?

D'un point de vue occidental, la construction d'un tunnel sous la cathédrale Notre-Dame de Paris serait inimaginable. Rappelons qu'en 1971, le gouvernement dirigé par Georges Pompidou avait décidé de créer la voie expresse rive gauche. La réalisation de la voie expresse rive droite, inaugurée le 22 décembre 1966, avait été décisive dans le projet de la voie expresse rive gauche. Néanmoins, le succès de cette dernière n'eut pas l'effet escompté sur la conception de la voie opposée. En effet, le projet de cette voie exécutée sur les quais bas de Seine, avait un tronçon central qui passait tout près de la cathédrale Notre-Dame de Paris. En outre, dès l'officialisation du projet de la rive gauche, des désaccords se manifestèrent et des associations virent le jour, afin de défendre le site de Notre-Dame de Paris. Ainsi, en 1971, fut fondé le comité de défense des berges de la Seine. Subséquemment, le mouvement se propagea, pour donner lieu à une manifestation, le 10 
juin 1972, au quai Saint-Bernard à Paris, contre la pollution et la création de la voie expresse rive gauche. Néanmoins, cette action citoyenne ne fit pas reculer le gouvernement Pompidou et le 25 septembre 1972, une maquette du projet fut présentée au ministre chargé des Affaires culturelles, Maurice Druon. L'action citoyenne redoubla alors d'efforts et en novembre 1973, une pétition circula contre la voie expresse rive gauche ${ }^{11}$. Face au mutisme du gouvernement, une autre manifestation eut lieu le 13 mars 1974. Ce problème de la voie expresse rive gauche prit tellement d'ampleur qu'il devint un sujet électoral de premier choix. À l'aube des élections présidentielles de 1974, Valérie Giscard d'Estaing et François Mitterrand prirent tous deux positions, le 25 avril et le 30 avril respectivement, en envoyant un courrier à l'Association pour la défense et l'embellissement du site de Notre-Dame de Paris. Une fois élu, Valéry Giscard d'Estaing suspendit les crédits pour la réalisation de la voie rive gauche. De plus, l'Élysée souhaita réexaminer les travaux du tronçon central, qui pose tant problème. Ainsi, en 1975, la voie express rive gauche fut supprimée.

6 Nous nous rendons donc compte que les principaux acteurs de la suppression de la voie expresse rive gauche furent les différentes associations, créées à l'occasion. De cette manière, la notion de défense du patrimoine parisien émergea au départ d'une partie de la population parisienne. À l'instar de l'exemple parisien, nous pouvons nous demander ce qu'il en est de la population de Rabat, concernant le tunnel des Oudayas. En interrogeant quelques habitants de la capitale marocaine sur la question, la vision du tunnel est sans équivoque. Ce dernier représente le nouvel emblème de la modernité de Rabat. D'un point de vue esthétique, il devient l'un des plus beaux joyaux de l'art industriel pour les habitants. Ainsi, nous nous demandons si la notion de patrimoine au Maroc est aujourd'hui la même que la notion de patrimoine en France. Est-ce ici que nous voyons la rupture avec la tradition de l'héritage français? Il faut avant tout revenir sur le fait que le Maroc est un pays émergent. De ce fait, les travaux d'infrastructures de grande envergure sont nécessaires pour le développement économique du pays et de sa capitale. Rabat se valorise, tant dans son modernisme que dans la préservation de son patrimoine passé.

7 Il faut rappeler que des spécialistes ont été engagés pour exécuter les restaurations de la Kasbah des Oudayas, ces dernières étant sous le contrôle de la direction du patrimoine du Royaume du Maroc. Dès 2009, les campagnes de consolidation furent entreprises. Les murs de soutènement de la Kasbah furent renforcés. De plus, des fissurations existantes au niveau de plusieurs bâtiments ont été réparées et leur base consolidée, afin de les stabiliser. Les premières interventions ont été réalisées sur le rempart nord-ouest, ce dernier portant des traces d'écoulements d'eau, et fut canalisé. D'autre part, au niveau du sémaphore, l'entrepôt Moulay Al Yazid a été débarrassé de ses annexes et de ses murs de clôture, pour retrouver son aspect d'origine. L'accès de la place du Sémaphore au niveau de la Sqala a été, quant à elle, réaménagé en établissant la réfection des escaliers. En outre, la réouverture des chemins de rondes a pu être réalisée afin d'offrir au public la jouissance d'un panorama exceptionnel, notamment sur le jardin et le front de mer. Ces restaurations montrent que le tunnel des Oudayas a permis une réhabilitation de la Kasbah, mais prouvent également que le Maroc tient à son patrimoine architectural. Cependant une question reste en suspend, lorsque Rabat en janvier 2011, propose sa candidature pour devenir patrimoine mondial de l'Unesco, le tunnel n'est pas encore achevé. Ce dernier peut-il poser un frein à cette accession? 


\section{NOTES}

1. 1 Bulletin Officiel du Protectorat de la République française, ${ }^{\circ} 5$, du 29 novembre 1912, p. 25-26.

2. 2 Bulletin Officiel du Protectorat de la République française, $n^{\circ} 5$, du 29 novembre 1912, p. 26.

3. 3 Dahir relatif à la conservation des monuments historiques et inscriptions, objets d'art et des antiquités, Bulletin Officiel du Protectorat de la République française, $n^{\circ} 70$, du 27 février 1914, p.126-129.

4. 4 Dahir déclarant comme monuments historiques certaines parties de la Kasba des Oudaya à Rabat, Bulletin Officiel du Protectorat de la République française, no 86, 19 juin 1914, p. 454.

5. 5 Maître artisan.

6. 6 Archives des la Bibliothèque nationale du royaume du Maroc (ABNRM), Rabat, fonds du protectorat français, série secrétariat général du protectorat, direction de l'Instruction publique, service des beaux-arts, antiquités et monuments historiques, carton $n^{\circ} \mathrm{G} 630$ relatif aux restaurations de la grande porte de la kasbah des Oudaïa, Rabat Maroc.

7. 7 ABNRM, Rabat, fonds du protectorat français, série secrétariat général du protectorat, direction de l'Instruction publique, service des beaux-arts, antiquités et monuments historiques, carton C 1978 relatif aux travaux de la medersa des Oudaïa dès 1916.

8. 8 ABNRM, Rabat, fonds du protectorat français, série secrétariat général du protectorat, direction de l'Instruction publique, service des beaux-arts, antiquités et monuments historiques, carton $n^{\circ} \mathrm{G} 630$ relatif aux restaurations de la grande porte de la kasbah des Oudaïa, Rabat, Maroc.

9. 9 Arrêté $n^{\circ}$ 503-91du 06/3/1992 - Bulletin officiel, $\mathrm{n}^{\circ} 4144,1^{\mathrm{er}}$ avril 1992.

10. Agence pour l'aménagement de la vallée du Bouregreg, Projet d'aménagement de la vallée du Bouregreg, Rabat, Agence pour l'aménagement de la vallée du Bouregreg, Mars 2003, 169 pages. Ce plan est disponible dans sa totalité sur le site www.bouregreg.com.

11. 11 Mathieu Flonneau, L'Automobile à la conquête de Paris chroniques illustrées, Paris, presses de l'École nationale des Ponts et chaussées, $287 \mathrm{p}$.

\section{RÉSUMÉS}

Dès 1912, le protectorat de France au Maroc inscrit une politique culturelle innovante dans le pays. En effet, en novembre 1912 un système législatif et une administration sont crées afin de protéger et restaurer le patrimoine bâti marocain. De ce fait, la Kasbah des Oudayas devient le premier monument Historique au Maroc le 6 juin 1914. Des restaurations s'engagent sur ce site historique, symbole de la ville de Rabat, dues à l'administration française mais également à des artisans marocains. Cet échange de savoir-faire nous laisse penser que l'expérience française au Maroc au niveau de la protection du patrimoine est la plus convaincante. En 1956, une fois l'indépendance du Maroc proclamée, Mohammed V conserva la plupart des lois culturelles déjà mises en place sous le protectorat. Dès les années 1980, la définition du patrimoine s'élargit et le «patrimoine naturel» ou encore le «patrimoine oral» sont considérés et protégés. Le 
développement économique du pays se fait rapidement et plusieurs politiques de grands travaux se succèdent. En 2006 Mohammed VI lance un projet d'infrastructure colossal pour la capitale du Maroc. Il est décidé de créer un second pont, un tramway et un tunnel dans la ville de Rabat. Le tunnel doit se situer sous la Kasbah des Oudayas, ce qui aurait pu se révéler un problème majeur dans l'histoire du patrimoine marocain, notamment si l'on se réfère à la création de la voie express rive gauche à Paris. Néanmoins, le tunnel des Oudayas reste au sein du Maroc l'une des plus belles infrastructures jamais réalisées.

By 1912, the protectorate of France in Morocco launched an innovative cultural policy in the country. Indeed, in November 1912 a legislative system and an administration were created to protect and restore the Moroccan built holdings. Therefore, Oudayas Kasbah became the first historical monument in Morocco on June 6th, 1914. Besides, the administration undertook the restoration of this Rabat's historic symbol, with the help of Moroccan craftsmen. This exchange of know-how makes us think that the French experience in Morocco in terms of heritage protection is the most convincing. In 1956, once the independence of Morocco proclaimed, the sovereign Mohammed V preserved most of the cultural laws initiated under the protectorate. From the 1980s, the definition of the heritage widened and the "natural heritage" or still the "oral heritage" were considered and protected. Besides, the country has known a quick economic development. In 2006, king Mohammed VI launched a massive infrastructure project for the Moroccan capital. Indeed, it was decided to build a second bridge, a tunnel and a tram for the city of Rabat. The tunnel had to go below the Oudayas Kasbah, which could prove to be a major problem in the history of Moroccan heritage, especially if one refers to the creation of the expressway left bank in Paris. However, the Oudayas's tunnel remains within Morocco one of the best infrastructures ever made.

Schon 1912 führte das französische Protektorat über Marokko eine innovative kulturelle Politik in diesem Lande ein. Tatsächlich entstanden im November 1912 ein gesetzgebendes Organ und entsprechende Verwaltungsdienste betreffs des Schutzes und der Restaurierung der gebauten Denkmäler. So wurde am 6. Juni 1914 die Kasbah der Oudayas das erste historische Denkmal in ar Marokko. Außerdem restaurierte die französische Verwaltung diesen sehenswürdigen historischen Platz - Symbol der Stadt Rabat - gemeinsam mit marokkanischen Handwerkskräften. An diesem Austausch von Fachkompetenzen lässt sich die Tragweite der französischen Erfahrung zur Denkmalerhaltung in Marokko auffällig erkennen. Aus diesen Gründen behielt König Mohammed V. nach der Unabhängigkeitserklärung 1956 die meisten der unter dem Protektorat entstandenen kulturellen Gesetze bei. Ab den Jahren 1980 erweiterte sich das Kulturerbe- Bewusstsein, indem Naturerbe und mündliches Kulturerbe anerkannt und unter Schutz gestellt wurden. Die rasche wirtschaftliche Entwicklung des Landes führte übrigens zu mehreren politischen Entscheidungen, bedeutsame Bauaufträge zu vergeben. 2006 initiierte König Mohammed VI. ein riesiges Infrastruktur-Projekt für die Hauptstadt Marokko, eine zweite Brücke, eine Straßenbahn und einen Tunnel. Der Bau des Tunnels unterhalb des Denkmals der Kasbah der Oudayas hätte für die Erhaltung des Status als marokkanisches Kulturerbe ein großes Risiko bergen können - siehe den umstrittenen Ausbau der Pariser Schnellstraße an dem linken Ufer der Seine. Der Status wurde aber keineswegs bestritten, und so erweist sich nun der Tunnel der Oudayas in Rabat als eine der schönsten je gebauten Infrastrukturen. 


\section{AUTEUR}

\section{NADÈGE THEILBORIE}

Nadège Theilborie, née en 1985, est titulaire d'une licence d'histoire de l'art et d'archéologie spécialité histoire de l'art à l'Université Paris-Sorbonne. En septembre 2009 elle entame un Master de recherche en histoire de l'art spécialité patrimoine moderne et contemporain à l'Université Paris-Sorbonne. Son travail de recherche intitulé. « La patrimonialisation du Maroc, l'exemple de Rabat » réalisé sous la tutelle de Barthélémy Jobert et Basile Baudez lui permet d'acquérir son diplôme de Master à l'université Paris-Sorbonne en septembre 2011. 